\title{
Reactive oxygen species generation by bovine blood neutrophils with different CXCR1 (ILRRA) genotype following Interleukin-8 incubation
}

\author{
Joren Verbeke ${ }^{1 *}$, Xanthippe Boulougouris ${ }^{2}$, Carolien Rogiers², Christian Burvenich², Luc Peelman ${ }^{3}$, \\ Bart De Spiegeleer ${ }^{4}$ and Sarne De Vliegher ${ }^{1}$
}

\begin{abstract}
Background: Associations between polymorphisms in the bovine CXCR1 gene, encoding the chemokine (C-X-C motif) receptor 1 (IL8RA), and neutrophil traits and mastitis have been described. In the present study, blood neutrophils were isolated from 20 early lactating heifers with different CXCR1 genotype at position 735 or 980 . The cells were incubated with different concentrations of recombinant bovine IL-8 (rbIL-8) for 2 or $6 \mathrm{~h}$ and stimulated with phorbol 12-myristate 13-acetate (PMA) or opsonized zymosan particles (OZP). Potential association between CXCR1 genotype and production of reactive oxygen species (ROS) was studied.

Results: Although on single nucleotide polymorphisms (SNPs) may potentially affect CXCR1 function, SNPs C.735C > G and c.980A > G showed no association with ROS production with or without incubation of rblL-8. Neutrophils incubated with rblL-8 for 2 or $6 \mathrm{~h}$ showed higher PMA- and lower OZP-induced ROS production compared to control without rblL-8.

Conclusions: In the present study no association could be detected between superoxide production by isolated bovine neutrophils during early lactation and CXCR1 gene polymorphism. IL-8 showed to possess inhibitory effects on ROS generation in bovine neutrophils.
\end{abstract}

Keywords: Bovine Neutrophil, Reactive Oxygen Species Generation, CXCR1 Polymorphism, Interleukin 8

\section{Background}

Intramammary infection induces a fast influx of blood neutrophils into the site of infection [1]. Activated neutrophils eliminate invading pathogens by phagocytosis and a diverse array of oxygen-dependent and oxygenindependent killing mechanisms. A powerful mechanism is the generation of reactive oxygen species (ROS) or superoxide [2]. It is widely accepted that neutrophils play a pivotal role in mammary gland immunity. Since 1990, an overwhelming amount of evidence has been generated of neutrophil dysfunction around parturition and early lactation with consequences on the defense of the mammary gland [3]. For example, although E.coli strains may influence the severity of infection, the

\footnotetext{
* Correspondence: Joren.Verbeke@UGent.be

${ }^{1} \mathrm{M}$-team and Mastitis and Milk Quality Research Unit, Department of Reproduction, Obstetrics, and Herd Health, Faculty of Veterinary Medicine, Ghent University, Salisburylaan, 133 Merelbeke, Belgium

Full list of author information is available at the end of the article
}

primary determinant of severity is the physiological state of the cow. Severity of experimentally induced E.coli mastitis during early lactation was tightly correlated with the pre-infection capacity of isolated blood neutrophils to generate ROS after zymosan and phorbol ester stimulation $[4,5]$ and their chemotactic response as well [6]. Interleukin 8 (IL-8), an important chemokine in the innate immune response of the mammary gland [3], enhances ROS generation [7], causes chemotaxis [8] and delays apoptosis [9] of isolated bovine blood neutrophils in vitro. Interleukin 8 priming of isolated human neutrophils for higher superoxide production was mediated through CXCR1 (IL8RA) and not through CXCR2 (IL8RB) $[10,11]$.

Many polymorphisms have been detected in the coding region of the bovine CXCR1 gene [12,13]. Single nucleotide polymorphism (SNP) c.735C > G (dbSNP ID: rs208795699) causes an amino acid change in the third 
intracellular loop (p.His245Glu) potentially affecting G-protein binding and signal transduction. Furthermore, c. $735 \mathrm{C}>\mathrm{G}$ was found to be in full linkage disequilibrium with SNPs c.37A $>\mathrm{T}$ (rs380621468), c. $38 \mathrm{~T}>\mathrm{A}(\mathrm{rs} 110296731)$ and c.68G > A (rs133273369) causing amino acid changes p.Ile13Tyr and p.Gly23Glu in the $\mathrm{N}$-terminus of CXCR1 known to have an important role in the first steps of binding IL-8 [13,14]. Associations between SNP c.735C > G and neutrophil functionality have been studied: blood neutrophils with genotype c.735GG showed a higher intracellular calcium release when stimulated with IL-8 and an increased ROS generation in response to PMA compared to neutrophils with genotype c.735CC (reviewed in [15]). Single nucleotide polymorphisms c.980A $>$ G (rs43323012) and c.995A > G (rs43323013) cause changes in the C-terminus (p.Lys327Arg and p.His332Arg) and might interfere with adaptin-2 binding and receptor internalization [12].

Previous research indicated an association between SNP c. $980 \mathrm{~A}>\mathrm{G}$ and likelihood of intramammary infection by major pathogens in early lactating heifers [13]. In the present study we wanted to know if CXCR1 gene polymorphism (SNPs c.735C > G and c.980A > G) could affect neutrophil functionality. Blood neutrophils, with different CXCR1 genotype, were isolated from 20 heifers during early lactation. ROS production as detected by chemiluminescence was measured following IL-8 incubation and stimulation with either PMA or opsonized zymosan particles (OZP). Freshly calved heifers were sampled because neutrophil functionality is reduced during this period [16].

We report the results of an association study between CXCR1SNPs c.735C > G and c.980A $>\mathrm{G}$ and blood neutrophil ROS. Additionally, we discuss the unexpected effect rbIL-8 had on neutrophil ROS depending on the stimulatory agent.

\section{Methods \\ Study design}

The experiment has been approved by the ethical committee of the Faculty of Veterinary Medicine, Ghent University (EC2013/190). Twenty Holstein heifers with different CXCR1 genotype were included from 5 different commercial dairy herds. Selected heifers were not siblings, had no history of diseases and all quarters were culture negative for major mastitis pathogens. Within $24 \mathrm{~h}$ after calving, neutrophils were isolated from blood and incubated with 0,40 or $400 \mathrm{ng} / \mathrm{ml}$ recombinant bovine IL-8 (rbIL-8) for 2 and $6 \mathrm{~h}$. Next, neutrophils were stimulated with PMA or OZP and ROS generation was measured by chemiluminescence. Finally associations between ROS generation and genotype, incubation time and rbIL- 8 concentration were statistically analyzed. The sample size $(n=20)$ was based on previous research demonstrating significant differences in ROS generation between 10 early and 10 mid lactating cows [16]. The incubation times were determined in a preliminary experiment in which blood neutrophils from 2 early lactating heifers were incubated for 2, 4, 6 and $18 \mathrm{~h}$ with 0,40 or $400 \mathrm{ng} / \mathrm{ml} \mathrm{rbIL-8.} \mathrm{A} \mathrm{differential} \mathrm{count} \mathrm{of} \mathrm{the} \mathrm{isolated}$ cells was performed to estimate the \% neutrophils. Viability of neutrophils was measured after isolation and after each incubation time by trypan blue exclusion.

\section{Bacteriological culture}

As mastitis can affect functionality of blood neutrophils [17], aseptic quarter milk samples were collected at the time of blood sampling and bacteriologically cultured. Ten $\mu \mathrm{L}$ of each sample was spread on blood-esculin and MacConkey's agar and incubated aerobically for 24-48 h at $37^{\circ} \mathrm{C}$. Bacteriological culture was performed according to National Mastitis Council (NMC) guidelines [18]. Four heifers were culture-positive in five quarters for major pathogens and excluded from the analysis. Staphylococcus aureus, esculin-positive cocci and Escherichia coli were isolated from 2 quarters of 1 heifers, 1 quarter of 1 heifer and 2 quarters of 2 heifers, respectively.

\section{CXCR1 genotype}

To include heifers with common and rare CXCR1 genotypes (e.g. c.980AA), a sufficient number of heifers were genotyped before calving. A blood sample was taken from 60 Holstein heifers belonging to 5 herds and having an expected calving date between January and June 2014. Genotype at SNPs c. $735 \mathrm{C}>\mathrm{G}$ and c.980A $>\mathrm{G}$ was determined using a fluorescent multiprobe PCR assay as previously described [19]. Efforts were made to include sufficient heifers with genotype c.980AA or c.980AG. Of the 20 heifers included in the final analysis, 7, 6 and 7 had genotype c.735CC, c.735CG and c.735GG, respectively. Three, 5 and 12 had genotype c.980AA, c.980AG and c.980GG, respectively.

\section{Reactive oxygen species assay}

Seventy-five $\mathrm{mL}$ blood was collected from the coccygeal vein using $8 \mathrm{~mL}$ Vacutainer tubes (Becton Dickinson, Erembodegem, Belgium) containing $150 \mu \mathrm{L}$ of EDTA as anticoagulant. Blood neutrophils were isolated within $1 \mathrm{~h}$ of collection by hypotonic lysis of red blood cells and Histopaque 1077/1119 gradient (Sigma-Aldrich, Bornem, Belgium) centrifugation according to Siemens et al. [20]. Cell concentration was measured in triplicate with a Bürker chamber.

Two hundred thousand blood neutrophils were suspended in $200 \mu \mathrm{L}$ of $1 \times$ Hank's balanced salt solution (HBSS; Gibco, Life technologies, Carlsbad, CA) supplemented with 0,40 or $400 \mathrm{ng}$ recombinant bovine IL-8 (rbIL-8; Kingfisher Biotech, Saint Paul, MN) per mL and incubated for 2 or $6 \mathrm{~h}$ at $37^{\circ} \mathrm{C}$ in $2 \mathrm{~mL}$ test tubes. 
Next, blood neutrophils were pelleted by centrifugation at $1000 \times g$ for $5 \mathrm{~min}$ and resuspended in $120 \mu \mathrm{l} 1 \times$ HBSS. Luminol $(0.30 \mathrm{mmol} / \mathrm{L}$ Sigma-Aldrich $)$ and PMA $(100 \mathrm{ng} / \mathrm{mL}$; Sigma-Aldrich) or OZP $(750 \mu \mathrm{g} / \mathrm{mL})$ were added to a final volume of $200 \mu \mathrm{L}$. Zymosan A (SigmaAldrich) was opsonized by washing the pellet with 60 and $30 \mathrm{~mL} 1 \times$ phosphate-buffered saline (PBS; Gibco) (centrifugation at $200 \times g$ for $10 \mathrm{~min}$ ) followed by $1 \mathrm{~h}$ incubation at $37^{\circ} \mathrm{C}$ in $5 \mathrm{~mL} 1 \times \mathrm{PBS}$ and $35 \mathrm{~mL}$ bovine serum and two additional washing steps with $30 \mathrm{~mL} 1 \times$ PBS (centrifugation at $200 \times g$ for $10 \mathrm{~min}$ ). Bovine serum was collected from the coccygeal vein of a healthy Holstein cow using $8 \mathrm{~mL}$ gel and clot activator tubes (Vacutest Kima, Piove di Sacco, Italy). Reactions of blood neutrophils primed by rbIL-8 were performed in duplicate. Chemiluminescence was measured every $60 \mathrm{sec}$ for 90 min with a luminometer ( $\operatorname{TriStar}^{2}$ LB 942 Multidetection Microplate Reader, Berthold Technologies, Bad Wildbad, Germany) and expressed in relative light units (RLU). Area under the curve (AUC) values (in $10^{6} \mathrm{RLU}^{*} \mathrm{~s}$ ) were calculated to analyze the total ROS generation whereas peak values (RLUmax; in $10^{3}$ RLU) and time of peak values (Tmax; in min) were saved in the dataset to study the kinetics of ROS generation [5].

\section{Statistical analysis}

Different linear mixed regression models (PROC MIXED, SAS 9.4, SAS Institute Inc.) were fit for AUC, RLUmax and Tmax after stimulation with PMA or OZP (6 outcome variables) and for SNP c.735C > G or c.980A $>$ G (12 models in total). Heifer was added as random effect to correct for clustering of multiple observations (6) per heifer (RANDOM statement). The models included heifers' genotype at position of the SNP, incubation ( 2 or $6 \mathrm{~h}$ ) and rbIL-8 (0, 40 or $400 \mathrm{ng} / \mathrm{ml}$ ) as categorical fixed effects. All two-way interactions between fixed effects were tested but removed from the models because they were non-significant $(P>0.05)$.

\section{Results}

\section{Preliminary experiment}

A differential count demonstrated 94.3\% [standard deviation (SD) $0.1 \%$ ] of the isolated cells to be neutrophils. The viability of the neutrophils was $100 \%$ immediately after isolation and decreased to 96\% (SD 1\%), 96\% (SD 2\%), 98\% (SD 1\%) and 81\% (SD 3\%) after 2, 4, 6 and $18 \mathrm{~h}$ isolation, respectively. Differences in viability between neutrophils incubated with 0,40 or $400 \mathrm{ng} / \mathrm{mL}$ rbIL-8 were small (data not shown). Because of the low viability and strongly diminished ROS generation after $18 \mathrm{~h} \mathrm{incu-}$ bation, neutrophils were incubated for 2 and $6 \mathrm{~h}$ (Figure 1). As expected [21], chemiluminescence increased fast after PMA stimulation with a clear peak and increased more gradually after OZP stimulation (Figure 1).

\section{Associations with ROS generation after PMA stimulation}

Single nucleotide polymorphisms c.735C $>\mathrm{G}$ and c.980A > G were not associated with AUC, RLUmax or Tmax $(P>0.05)$ (Table 1). Incubation was associated with AUC, RLUmax and Tmax. Blood neutrophils incubated for $6 \mathrm{~h}$ showed higher AUC, RLUmax and Tmax values compared to blood neutrophils incubated for $2 \mathrm{~h}$ $(P<0.05)$. Concentration of rbIL-8 was associated with AUC and Tmax $(P<0.01)$ and not with RLUmax $(P=0.17)$. Blood neutrophils incubated with 40 or $400 \mathrm{ng} / \mathrm{mL}$ showed higher AUC and Tmax values compared to blood neutrophils incubated without rbIL-8.

\section{Associations with ROS generation after OZP stimulation}

Single nucleotide polymorphisms c.735C $>\mathrm{G}$ and c.980A $>$ G were not associated with AUC, RLUmax or Tmax $(P>0.05)$ (Table 2). Incubation was associated with AUC and RLUmax and not with Tmax. Blood neutrophils incubated for $6 \mathrm{~h}$ showed higher AUC and RLUmax values compared to blood neutrophils incubated for $2 \mathrm{~h}(P<0.05)$. Concentration of rbIL-8 was associated with AUC and RLUmax $(P<0.01)$ and not with Tmax $(P=0.89)$. Blood neutrophils incubated with 40 or $400 \mathrm{ng} / \mathrm{ml}$ showed lower AUC and Tmax values compared to blood neutrophils incubated without rbIL8. Differences were mainly in neutrophils incubated with rbIL-8 at a concentration of $400 \mathrm{ng} / \mathrm{mL}$, AUC values were smaller.

\section{Discussion}

Research on genetic polymorphisms enlarges our knowledge on mammary gland immunity and helps us to understand why certain cows are more mastitis resistant than others [15]. Because of the important function of CXCR1 in the innate immunity of the mammary gland $[8,22]$ and a quantitative trait locus for clinical mastitis in this region of the bovine genome [23], CXCR1 polymorphisms form interesting study objects. In this study, an in vitro model was used to analyze the effect of CXCR1 SNP on neutrophil functionality in a sample population of freshly calved heifers. Associations between CXCR1 genotype and neutrophil ROS generation after rbIL-8 incubation and stimulation with PMA or OZP were studied in detail.

The association between SNP c.735C > G and PMAinduced ROS generation reported by Rambeaud et al. (2006) could not be confirmed in our model. In contrast to the previously demonstrated higher ROS generation [9], we observed numerically lower AUC values in c.735GG neutrophils compared to c.735CC neutrophils. Additionally, no significant interaction effects between c. $735 \mathrm{C}>\mathrm{G}$ and rbIL-8 concentration were observed. Based on previous research [13], we hypothesized a higher ROS generation and response to rbIL-8 in c.980AG 


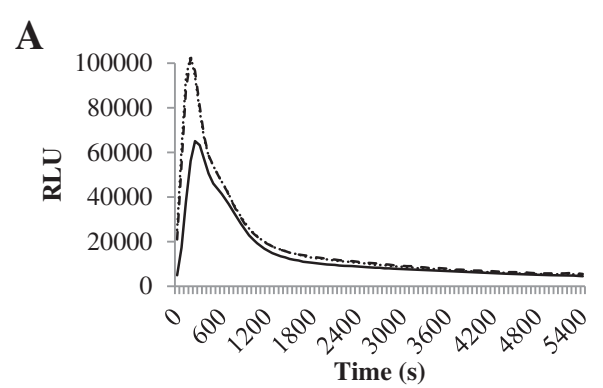

C

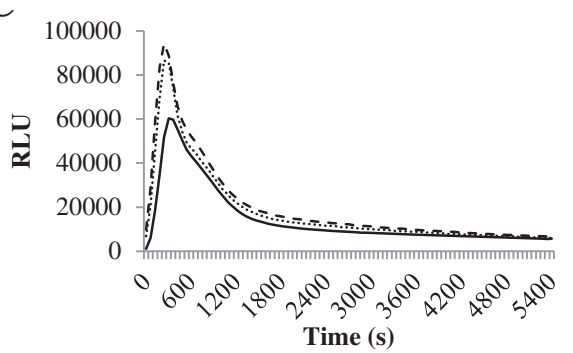

E

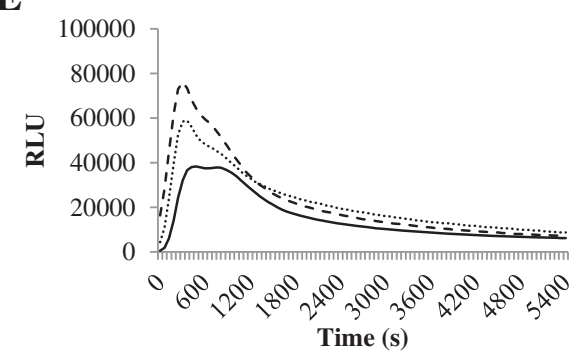

G

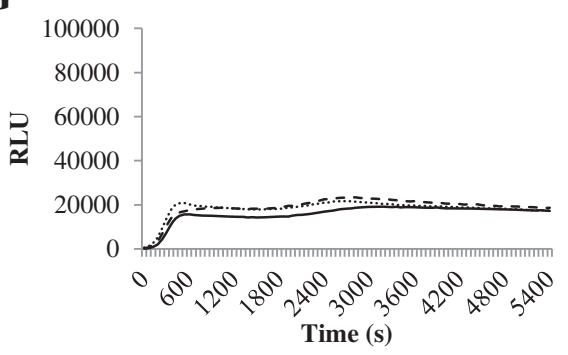

B

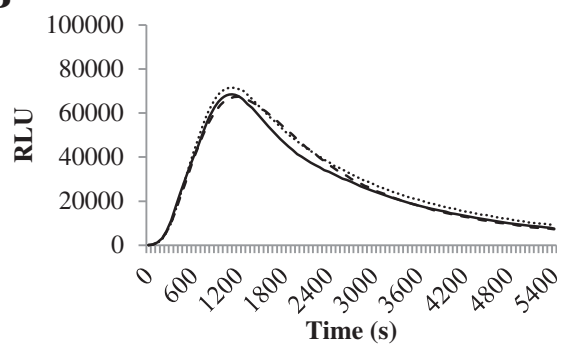

D

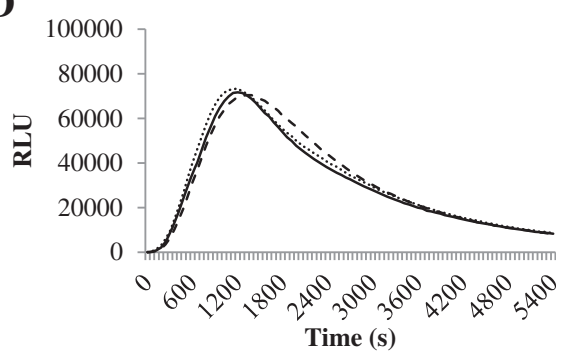

F

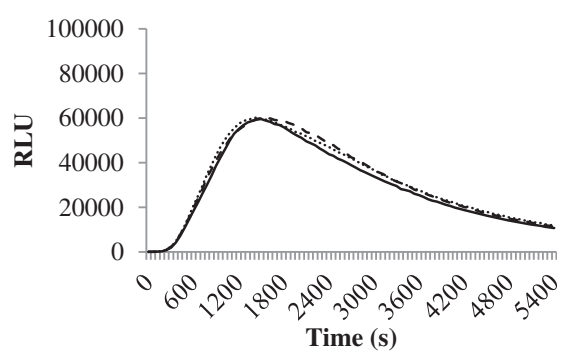

H

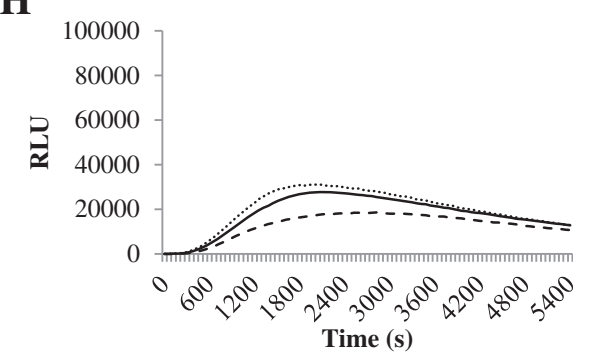

Figure 1 Effect of Interleukin-8 on reactive oxygen species generation of blood neutrophils.Preliminary experiment studying the reactive oxygen species (ROS) generation of blood neutrophils isolated from 2 early lactating dairy cows and incubated with 0 (- $\longrightarrow$ ), $40(\ldots)$ or 400 (- - -) ng recombinant bovine interleukin-8 (rbll-8) per $\mathrm{mL}$ for 2 (A and $\mathbf{B}), 4$ (C and $\mathbf{D}), 6$ (E and $\mathbf{F}$ ) or 18 (G and $\mathbf{H}$ ) h measured using luminol chemiluminescence [expressed in relative light units (RLU)]. Prior to measurement, neutrophils were stimulated with phorbol 12-myristate 13-acetate (A, C, E and $\mathbf{G})$ or opsonized zymosan particles (B, D, F and $\mathbf{H})$.

compared to c.980GG neutrophils. However, neither c. $980 \mathrm{~A}>\mathrm{G}$ nor the interaction between rbIL-8 concentration and c.980A > G were associated with AUC, RLUmax or Tmax of PMA- or OZP-induced ROS generation in our model. Despite potential effects on ligand binding, signal transduction and internalization of CXCR1 [12], no interaction between CXCR1 SNPs and rbIL-8 incubation could be demonstrated. Human CXCR1 but not hCXCR2 was found to be important for activation of ROS [24]. Latter functional differences were partly attributed to amino acid sequence differences in the C-terminus causing a faster receptor phosphorylation and internalization of hCXCR2 compared to hCXCR1 [25-27]. In contrast to hCXCR1 and hCXCR2, the C-terminus of both bovine IL-8R show identical amino acid sequences [28]. Hence, activation of neutrophils could be mediated by both receptors and functional effects caused by CXCR1 SNP might be compensated by a fully functional CXCR2, explaining similar rbIL-8 responses on ROS across CXCR1 genotypes in our study.

Several studies demonstrated a priming effect of IL-8 on ROS generation in human neutrophils $[10,29,30]$. 
Table 1 Statistical analysis of reactive oxygen species generation by blood neutrophils following phorbol 12-myristate 13-acetate stimulation

\begin{tabular}{|c|c|c|c|c|c|c|c|c|c|c|c|}
\hline \multirow{2}{*}{ Polymorphism ${ }^{1}$} & \multirow{2}{*}{ Fixed effect } & \multirow{2}{*}{$\mathrm{n}^{2}$} & \multicolumn{3}{|l|}{$A U C^{3}$} & \multicolumn{3}{|c|}{ RLUmax $^{4}$} & \multicolumn{3}{|c|}{$\operatorname{Tmax}^{5}$} \\
\hline & & & $\beta^{6}$ & $\mathrm{SE}^{7}$ & $P^{8}$ & $\overline{\beta^{6}}$ & $\mathrm{SE}^{7}$ & $P^{8}$ & $\beta^{6}$ & $\mathrm{SE}^{7}$ & $P^{8}$ \\
\hline \multirow[t]{12}{*}{ c. $735 C>G$} & Intercept & & 71 & 8 & & 37 & 6 & & 9.2 & 1.0 & \\
\hline & Genotype & & & & 0.69 & & & 0.94 & & & 0.47 \\
\hline & c.735CC & 7 & Ref. $^{9}$ & $\ldots$ & & Ref. $^{9}$ & $\ldots$ & & Ref. $^{9}$ & $\ldots$ & \\
\hline & c.735CG & 6 & -6 & 11 & & -3 & 9 & & 0.3 & 1.3 & \\
\hline & c.735GG & 7 & -9 & 11 & & -1 & 8 & & -1.2 & 1.3 & \\
\hline & Incubation & & & & $<0.01$ & & & $<0.01$ & & & $<0.05$ \\
\hline & $2 \mathrm{~h}$ & & Ref. $^{9}$ & $\cdots$ & & Ref. $^{9}$ & $\ldots$ & & Ref. $^{9}$ & $\ldots$ & \\
\hline & $6 \mathrm{~h}$ & & 11 & 2 & & 4 & 1 & & 1.2 & 0.5 & \\
\hline & rblL-8 & & & & $<0.01$ & & & 0.17 & & & $<0.01$ \\
\hline & $0 \mathrm{ng} / \mathrm{ml}$ & & Ref. $^{9}$ & $\cdots$ & & Ref. $^{9}$ & $\cdots$ & & Ref. $^{9}$ & $\cdots$ & \\
\hline & $40 \mathrm{ng} / \mathrm{ml}$ & & 7 & 2 & & -3 & 2 & & 3.1 & 0.6 & \\
\hline & 400 ng/ml & & 5 & 2 & & -2 & 2 & & 3.1 & 0.6 & \\
\hline \multirow[t]{12}{*}{$c .980 A>G$} & Intercept & & 66 & 11 & & 29 & 9 & & 8.9 & 1.5 & \\
\hline & Genotype & & & & 0.45 & & & 0.56 & & & 0.78 \\
\hline & C.980AA & 3 & Ref. $^{9}$ & $\cdots$ & & Ref. $^{9}$ & $\cdots$ & & Ref. $^{9}$ & $\cdots$ & \\
\hline & C.980AG & 5 & 10 & 14 & & 12 & 11 & & 0.7 & 1.8 & \\
\hline & c.980GG & 12 & -4 & 13 & & 5 & 10 & & -0.2 & 1.6 & \\
\hline & Incubation & & & & $<0.01$ & & & $<0.01$ & & & $<0.05$ \\
\hline & $2 \mathrm{~h}$ & & Ref. $^{9}$ & $\ldots$ & & Ref. $^{9}$ & $\ldots$ & & Ref. $^{9}$ & $\cdots$ & \\
\hline & $6 \mathrm{~h}$ & & 11 & 2 & & 4 & 1 & & 1.2 & 0.5 & \\
\hline & rblL-8 & & & & $<0.01$ & & & 0.17 & & & $<0.01$ \\
\hline & $0 \mathrm{ng} / \mathrm{ml}$ & & Ref. $^{9}$ & $\ldots$ & & Ref. $^{9}$ & $\ldots$ & & Ref. $^{9}$ & $\ldots$ & \\
\hline & 40 ng/ml & & 7 & 2 & & -3 & 2 & & 3.1 & 0.6 & \\
\hline & $400 \mathrm{ng} / \mathrm{ml}$ & & 5 & 2 & & -2 & 2 & & 3.1 & 0.6 & \\
\hline
\end{tabular}

${ }^{1}$ Linear mixed regression models describing the association between reactive oxygen species generation by blood neutrophils and CXCR1 polymorphisms

c. $735 \mathrm{C}>\mathrm{G}$ and c.980A $>\mathrm{G}$, respectively. Neutrophils were incubated with 0, 40 or $400 \mathrm{ng}$ recombinant bovine interleukin 8 per mL for 2 or $6 \mathrm{~h}$ and stimulated

with phorbol 12-myristate 13-acetate,

${ }^{2}$ Number of heifers,

${ }^{3}$ Area under the curve values in $10^{6} \mathrm{RLU}$ (relative light units) * $\mathrm{s}$,

${ }^{4}$ Peak values in $10^{3}$ RLU.

${ }^{5}$ Time of peak values in min,

${ }^{6}$ Regression coefficient

${ }^{7}$ Standard error,

${ }^{8}$ Overall $P$-value of the fixed effect,

${ }^{9}$ Reference,

All two-way interactions between the fixed effects were non-significant $(P>0.05)$ and removed from the model.

Enhancement of ROS generation already occurred after 5 min of IL-8 incubation [29] and was explained by intracellular calcium mobilization [30] and by activation of phospholipase D (PLD) [10], protein kinase C- $\varepsilon$ (PKC- - ) [25] and phospholipase $\mathrm{A}_{2}$ [29]. To the best of our knowledge, Mitchell et al. [7] were the only to study the priming effect of IL-8 on bovine neutrophils. Intracellular ROS generation was measured by 2,7-Dichlorodihydrofluorecein diacetate (H2DCFDA) flow cytometry. Incubation with rbIL- 8 for $18 \mathrm{~h}$ enhanced the Mannheimia haemolytica-induced ROS generation whereas incubation for 30 or $60 \mathrm{~min}$ had little effect [7]. In our study,
ROS generation was measured using luminol chemiluminescence. This assay has the benefit that both intraand extracellular ROS generation are measured and allows for measurement over time [31]. Neutrophils were exposed to the same concentrations of rbIL- 8 as in the study of Mitchell et al. [7] but for only 2 or $6 \mathrm{~h}$. We opted not to incubate for $18 \mathrm{~h}$ because our preliminary experiments showed a reduced viability and a strongly diminished ROS generation after such a long period. Associations were detected between rbIL-8 concentration and ROS generation indicating the presence of a functional IL-8 receptor on the isolated blood neutrophils. In 
Table 2 Statistical analysis of reactive oxygen species generation by blood neutrophils following opsonized zymosan particles stimulation

\begin{tabular}{|c|c|c|c|c|c|c|c|c|c|c|c|}
\hline \multirow{2}{*}{ Polymorphism ${ }^{1}$} & \multirow{2}{*}{ Fixed effect } & \multirow{2}{*}{$\mathrm{n}^{2}$} & \multicolumn{3}{|c|}{$A_{U C}{ }^{3}$} & \multicolumn{3}{|c|}{ RLUmax ${ }^{4}$} & \multicolumn{3}{|c|}{$\operatorname{Tmax}^{5}$} \\
\hline & & & $\beta^{6}$ & $\mathrm{SE}^{7}$ & $P^{8}$ & $\overline{\beta^{6}}$ & $\mathrm{SE}^{7}$ & $P^{8}$ & $\overline{\beta^{6}}$ & $\mathrm{SE}^{7}$ & $P^{8}$ \\
\hline \multirow[t]{12}{*}{ c. $735 C>G$} & Intercept & & 253 & 32 & & 69 & 10 & & 31.7 & 4.0 & \\
\hline & Genotype & & & & 0.40 & & & 0.82 & & & 0.22 \\
\hline & c.735CC & 7 & Ref. ${ }^{9}$ & $\cdots$ & & Ref. $^{9}$ & $\cdots$ & & Ref. $^{9}$ & $\ldots$ & \\
\hline & c.735CG & 6 & -36 & 47 & & -6 & 14 & & -3.6 & 5.3 & \\
\hline & c.735GG & 7 & -63 & 45 & & -9 & 14 & & -9.2 & 5.1 & \\
\hline & Incubation & & & & $<0.01$ & & & $<0.01$ & & & 0.54 \\
\hline & $2 \mathrm{~h}$ & & Ref. $^{9}$ & $\cdots$ & & Ref. $^{9}$ & $\cdots$ & & Ref. $^{9}$ & $\cdots$ & \\
\hline & $6 \mathrm{~h}$ & & 20 & 7 & & 5 & 2 & & 1.2 & 1.9 & \\
\hline & rblL-8 & & & & $<0.01$ & & & $<0.01$ & & & 0.89 \\
\hline & $0 \mathrm{ng} / \mathrm{ml}$ & & Ref. $^{9}$ & $\cdots$ & & Ref. $^{9}$ & $\cdots$ & & Ref. $^{9}$ & $\cdots$ & \\
\hline & $40 \mathrm{ng} / \mathrm{ml}$ & & -6 & 8 & & -3 & 2 & & -0.6 & 2.3 & \\
\hline & 400 ng/ml & & -26 & 8 & & -8 & 2 & & 0.5 & 2.3 & \\
\hline \multirow[t]{12}{*}{ c. $980 A>G$} & Intercept & & 248 & 48 & & 63 & 15 & & 32.9 & 6.0 & \\
\hline & Genotype & & & & 0.32 & & & 0.58 & & & 0.44 \\
\hline & c.980AA & 3 & Ref. $^{9}$ & $\ldots$ & & Ref. $^{9}$ & $\ldots$ & & Ref. $^{9}$ & $\ldots$ & \\
\hline & c.980AG & 5 & 13 & 61 & & 12 & 18 & & -3.6 & 7.3 & \\
\hline & c.980GG & 12 & -51 & 54 & & -2 & 16 & & -7.8 & 6.4 & \\
\hline & Incubation & & & & $<0.01$ & & & $<0.01$ & & & 0.54 \\
\hline & $2 \mathrm{~h}$ & & Ref. $^{9}$ & $\ldots$ & & Ref. $^{9}$ & $\ldots$ & & Ref. $^{9}$ & $\ldots$ & \\
\hline & $6 \mathrm{~h}$ & & 20 & 7 & & 5 & 2 & & 1.2 & 1.9 & \\
\hline & rblL-8 & & & & $<0.01$ & & & 0.17 & & & 0.89 \\
\hline & $0 \mathrm{ng} / \mathrm{ml}$ & & Ref. $^{9}$ & $\cdots$ & & Ref. $^{9}$ & $\cdots$ & & Ref. $^{9}$ & $\cdots$ & \\
\hline & 40 ng/ml & & -6 & 8 & & -3 & 2 & & -0.6 & 2.3 & \\
\hline & 400 ng/ml & & -26 & 8 & & -8 & 2 & & 0.5 & 2.3 & \\
\hline
\end{tabular}

${ }^{1}$ Linear mixed regression models describing the association between reactive oxygen species generation by blood neutrophils and $C X C R 1$ polymorphisms

c.735C > G and c.980A > G, respectively. Neutrophils were incubated with 0,40 or 400 ng recombinant bovine interleukin 8 per mL for 2 or $6 \mathrm{~h}$ and stimulated

with opsonized zymosan particles,

${ }^{2}$ Number of heifers,

${ }^{3}$ Area under the curve values in $10^{6} \mathrm{RLU}$ (relative light units) ${ }^{*} \mathrm{~S}$,

${ }^{4}$ Peak values in $10^{3} \mathrm{RLU}$

${ }^{5}$ Time of peak values in $\mathrm{min}$

${ }^{6}$ Regression coefficient,

${ }^{7}$ Standard error,

${ }^{8}$ Overall $P$-value of the fixed effect,

${ }^{9}$ Reference,

All two-way interactions between the fixed effects were non-significant $(P>0.05)$ and removed from the model.

contrast to research on human neutrophils [29,30], IL-8 also had inhibitory effects on neutrophils ROS generation in our model. Incubation with rbIL-8 had a positive effect on the total PMA-induced ROS generation but a negative effect on the total OZP-induced ROS generation. The stimulatory agent dependent effect could be explained by differences in the pathways of ROS generation by PMA and OZP [32]. Incubation with rbIL-8 might have simultaneously activated components of the pathway induced by PMA (e.g. PKC) while inhibiting components of the pathway induced by OZP (e.g. calcium mobilization).

\section{Conclusions}

In conclusion, no differences in PMA- or OZP-induced ROS generation were detected in blood neutrophils isolated from early lactating heifers with different CXCR1 c.735C > G and c.980A > G genotypes. The inhibitory effects of rbIL-8 on neutrophil ROS generation suggest a complex interaction between IL-8 and ROS generation in bovine neutrophils.

\section{Abbreviations}

AUC: Area under the curve; CXCR1: Chemokine (C-X-C motif) receptor 1; IL-8: Interleukin 8; IL8RA: Interleukin 8 receptor A; NMC: National mastitis council; OZP: Opsonized zymosan particles; PMA: Phorbol 12-myristate 
13-acetate; rblL-8: Recombinant bovine interleukin 8; RLU: Relative light units; RLUmax: Peak value; ROS: Reactive oxygen species; SCC: Somatic cell count; SNP: Single nucleotide polymorphism; Tmax: Time of peak value.

\section{Competing interests}

The authors declare that they have no competing interests.

\section{Authors' contributions}

$J \vee, X B, C B, L P$ and SDV designed the experiment. JV, XB and CR collected milk and blood samples. JV genotyped animals. CR isolated blood neutrophils. $J \mathrm{~V}, \mathrm{XB}$ and $\mathrm{CR}$ conducted the chemiluminescence assay. JV analyzed data and drafted the manuscript. XB, CB, LP, BDS and SDV gave critical comments on the manuscript. All authors read and approved the final manuscript.

\section{Acknowledgements}

This research was financed by a PhD grant ( $\left.n^{\circ} 101206\right)$ by the Agency for Innovation by Science and Technology in Flanders (IWT Vlaanderen). The authors wish to thank the participating dairy producers and bovine practitioner Raf Deconinck

\section{Author details}

${ }^{1}$ M-team and Mastitis and Milk Quality Research Unit, Department of Reproduction, Obstetrics, and Herd Health, Faculty of Veterinary Medicine, Ghent University, Salisburylaan, 133 Merelbeke, Belgium. ²Department of Comparative Physiology and Biometrics, Faculty of Veterinary Medicine, Ghent University, Salisburylaan, 133 Merelbeke, Belgium. ${ }^{3}$ Animal Genetics Laboratory, Department of Nutrition, Genetics, and Ethology, Faculty of Veterinary Medicine, Ghent University, Heidestraat, 19 Merelbeke, Belgium. ${ }^{4}$ Laboratory of Drug Quality \& Registration, Department of Pharmaceutical Analysis, Faculty of Pharmaceutical Sciences, Ghent University, Ottergemsesteenweg, 460 Ghent, Belgium.

\section{Received: 25 September 2014 Accepted: 26 April 2015 Published online: 06 May 2015}

\section{References}

1. Rainard P, Riollet C. Innate immunity of the bovine mammary gland. Vet Res. 2006:37:369-400.

2. Mehrzad J, Duchateau L, Burvenich C. Phagocytic and bactericidal activity of blood and milk-resident neutrophils against staphylococcus aureus in primiparous and multiparous cows during early lactation. Vet Microbiol. 2009:134:106-12.

3. Paape M, Mehrzad J, Zhao X, Detilleux J, Burvenich C. Defense of the bovine mammary gland by polymorphonuclear neutrophil leukocytes. J Mammary Gland Biol Neoplasia. 2002;7:109-21.

4. Heyneman R, Burvenich C, Vercauteren R. Interaction between the respiratory burst activity of neutrophil leukocytes and experimentally induced Escherichia coli mastitis in cows. J Dairy Sci. 1990;73:985-94.

5. Mehrzad J, Duchateau L, Burvenich C. High milk neutrophil chemiluminescence limits the severity of bovine coliform mastitis. Vet Res. 2005:36:101-16.

6. Kremer WD, Noordhuizen-Stassen EN, Grommers FJ, Daemen AJ, Henricks PA, Brand $A$, et al. Preinfection chemotactic response of blood polymorphonuclear leukocytes to predict severity of Escherichia coli mastitis. J Dairy Sci. 1993;76:1568-74

7. Mitchell GB, Albright BN, Caswell JL. Effect of interleukin-8 and granulocyte colony-stimulating factor on priming and activation of bovine neutrophils. Infect Immun. 2003;71:1643-9.

8. Barber MR, Yang TJ. Chemotactic activities in nonmastitic and mastitic mammary secretions: Presence of interleukin-8 in mastitic but not nonmastitic secretions. Clin Diagn Lab Immunol. 1998:5:82-6.

9. Rambeaud M, Clift R, Pighetti GM. Association of a bovine CXCR2 gene polymorphism with neutrophil survival and killing ability. Vet Immunol Immunopathol. 2006;111:231-8.

10. Jones SA, Wolf M, Qin SX, Mackay CR, Baggiolini M. Different functions for the interleukin 8 receptors (IL-8R) of human neutrophil leukocytes: NADPH oxidase and phospholipase D are activated through IL-8R1 but not IL-8R2. Proc Natl Acad Sci U S A. 1996:93:6682-6.

11. Jones SA, Dewald B, ClarkLewis I, Baggiolini M. Chemokine antagonists that discriminate between interleukin-8 receptors - Selective blockers of CXCR2. J Biol Chem. 1997;272:16166-9.
12. Pighetti GM, Kojima CJ, Wojakiewicz L, Rambeaud M. The bovine CXCR1 gene is highly polymorphic. Vet Immunol Immunopathol. 2012;145:464-70.

13. Verbeke J, Piepers S, Peelman $\sqcup$, Van Poucke M, De Vliegher S. Pathogen-group specific association between CXCR1 polymorphisms and subclinical mastitis in dairy heifers. J Dairy Res. 2012;79:341-51

14. Liou JW, Chang FT, Chung Y, Chen WY, Fischer WB, Hsu HJ: In Silico analysis reveals sequential interactions and protein conformational changes during the binding of chemokine CXCL-8 to its receptor CXCR1. Plos One 2014, 9:e94178

15. Pighetti GM, Elliott AA. Gene polymorphisms: the keys for marker assisted selection and unraveling core regulatory pathways for mastitis resistance. J Mammary Gland Biol Neoplasia. 2011;16:421-32.

16. Mehrzad J, Dosogne H, Meyer E, Heyneman R, Burvenich C. Respiratory burst activity of blood and milk neutrophils in dairy cows during different stages of lactation. J Dairy Res. 2001;68:399-415.

17. Mehrzad J, Dosogne H, Meyer E, Burvenich C. Local and systemic effects of endotoxin mastitis on the chemiluminescence of milk and blood neutrophils in dairy cows. Vet Res. 2001;32:131-44.

18. National Mastitis Council: Laboratory Handbook on Bovine Mastitis. Madison, Wl: National Mastitis Counc. Inc. Wisconsin: The National Mastitis Council; 1999

19. Verbeke J, Van Poucke M, Peelman L, Piepers S, De Vliegher S. Associations between $C X C R 1$ polymorphisms and pathogen-specific clinical mastitis, test-day somatic cell count and test-day milk yield. J Dairy Sci. 2014;97:7927-39.

20. Siemens DW, Schepetkin IA, Kirpotina LN, Lei B, Quinn MT. Neutrophil isolation from nonhuman species. Methods Mol Biol. 2007:138:21-34.

21. Lieberman MM, Sachanandani DM, Pinney CA. Comparative study of neutrophil activation by chemiluminescence and flow cytometry. Clin Diagn Lab Immunol. 1996;3:654-62

22. Caswell JL, Middleton DM, Gordon JR. Production and functional characterization of recombinant bovine interleukin-8 as a specific neutrophil activator and chemoattractant. Vet Immunol Immunopathol. 1999;67:327-40.

23. Sodeland M, Kent MP, Olsen HG, Opsal MA, Svendsen M, Sehested $E_{1}$ et al. Quantitative trait loci for clinical mastitis on chromosomes 2, 6, 14 and 20 in Norwegian Red cattle. Anim Genet. 2011:42:457-65.

24. Stillie R, Faroog SM, Gordon JR, Stadnyk AW. The functional significance behind expressing two IL-8 receptor types on PMN. J Leukoc Biol. 2009;86:529-43.

25. Nasser MW, Marjoram RJ, Brown SL, Richardson RM. Cross-desensitization among CXCR1, CXCR2, and CCR5: Role of protein kinase C-epsilon. J Immunol. 2005;174:6927-33.

26. Richardson RM, Marjoram RJ, Barak LS, Snyderman R. Role of the cytoplasmic tails of CXCR1 and CXCR2 in mediating leukocyte migration, activation, and regulation. J Immunol. 2003:170:2904-11.

27. Richardson RM, Pridgen BC, Haribabu B, Ali H, Snyderman R. Differential cross-regulation of the human chemokine receptors CXCR1 and CXCR2 - Evidence for time-dependent signal generation. J Biol Chem. 1998:273:23830-6.

28. Lahouassa H, Rainard P, Caraty A, Riollet C. Identification and characterization of a new interleukin-8 receptor in bovine species. Mol Immunol. 2008;45:1153-64.

29. Daniels RH, Finnen MJ, Hill ME, Lackie JM. Recombinant human monocyte II-8 primes Nadph-oxidase and Phospholipase-A2 activation in human neutrophils. Immunology. 1992;75:157-63.

30. Wozniak A, Betts WH, Murphy GA, Rokicinski M. Interleukin-8 primes human neutrophils for enhanced superoxide anion production. Immunology. 1993:79:608-15.

31. Rinaldi M, Moroni P, Paape MJ, Bannerman DD. Evaluation of assays for the measurement of bovine neutrophil reactive oxygen species. Vet Immunol Immunopathol. 2007;115:107-25.

32. Maridonneau-Parini I, Tringale SM, Tauber Al. Identification of distinct activation pathways of the human neutrophil Nadph-oxidase. J Immunol. 1986;137:2925-9. 